\title{
Actividad físico-deportiva, autoconcepto físico y satisfacción con la vida
}

\author{
Eider Goñi y Guillermo Infante \\ Universidad del País Vasco (España)
}

\begin{abstract}
La relación entre la actividad físico-deportiva y el autoconcepto físico está bien documentada. De otro lado, diversos estudios respaldan el hecho de que la actividad físico-deportiva está vinculada a la satisfacción con la vida, relación que algunos autores defienden que está mediada por el autoconcepto físico. Con el objeto de comprobar de qué manera están relacionadas estas tres variables, en este estudio participan 408 personas (163 hombres; 245 mujeres) de entre 23 y 65 años $(M=44.82 ; D T=13.44)$; se les clasifica en sedentarias (37.5\%), moderadamente activas (35.4\%), activas (16\%) y muy activas (11\%), en función del gasto metabólico (expresado en METs) calculado a partir de la frecuencia $\times$ la duración $\times$ el valor de la actividad física que desarrollan. Los resultados permiten identificar relaciones positivas de la actividad físico-deportiva con la autopercepción física y con la satisfacción con la vida en el siguiente sentido: a mayor práctica de actividad físico-deportiva, mejor autoconcepto y mejores niveles de satisfacción con la vida.
\end{abstract}

Palabras clave: Actividad físico-deportiva, METs, autoconcepto físico, satisfacción con la vida.

Physical-sporting activity, physical self-concept and satisfaction with life. The relationship between physical-sporting activity and physical self-concept has been well documented. Furthermore, diverse studies also support the finding that physical-sporting activity is linked to satisfaction with life, a relationship which some authors believe to be mediated by physical self-concept. This study aims to verify the way in which these three variables are related. Participants were 408 adults (163 men and 245 women) aged between 23 and $65(M=44.82 ; S D=13.44)$, classified into sedentary $(37.5 \%)$, moderately active $(35.4 \%)$, active $(16 \%)$ and very active (11\%), depending on metabolic expenditure (expressed as METs) calculated on the basis of frequency $x$ duration $x$ value of the physical activity carried out. The results reveal positive associations between physical-sporting activity and both physical self-concept and satisfaction with life, in the following sense: the more physical-sporting activity engaged in, the better the subject's self-concept and the greater their satisfaction with life.

Key words: Physical-sporting activity, METs, physical self-concept, satisfaction with life.

Correspondencia: Eider Goñi Palacios. Universidad del País Vasco. Escuela Universitaria de Magisterio. Plaza de Oñati, 1. C.P. 20018. San Sebastián (España). E-mail: eider.goni@ehu.es 
Con el advenimiento de las sociedades postindustriales el estilo de vida de las personas se ha transformado de manera sustancial. Estos cambios han repercutido en la salud de los individuos: a) en el ámbito anatómico-fisiológico han surgido las denominadas enfermedades hipocinéticas que tienen relación estrecha con la falta de movimiento (Montil y Jiménez, 2006); b) en el ámbito psicológico el estrés que conlleva este tipo de sociedades ha generado alteraciones en el estado psicológico del individuo, tales como la depresión y la ansiedad (Biddle, Fox y Boutcher, 2000). En el periodo adulto a estas alteraciones psicosomáticas se le añaden los problemas derivados del proceso de envejecimiento. Este periodo de la vida está asociado con la aparición de deficiencias crónicas y comorbilidad. En resumen, todos estos factores resultan en reducciones de la calidad de vida de las personas y sus consecuencias influyen en la salud física y mental de los individuos.

En línea con la definición dada por la OMS sobre la salud, desde la psicología positiva se entiende que uno de los elementos clave de la misma es el bienestar psicológico de la persona (Fierro, 2007). El estudio del bienestar, así mismo, se puede clasificar en dos grandes tradiciones (Ryan y Deci, 2001): una relacionada fundamentalmente con la felicidad (bienestar hedónico) y otra ligada al desarrollo del potencial humano (bienestar eudaemónico).

A su vez, cabe distinguir en la trayectoria investigadora del bienestar dos grandes etapas. Inicialmente en el estudio de este constructo el aspecto central es la propia evaluación que la persona hace de su vida (Diener, 2000), la cual dará lugar a la estructura del bienestar según los diferentes dominios de la vida que son evaluados.

En un segundo momento, el interés investigador se centra en el estudio de los procesos que subyacen al bienestar (Cuadra y Florenzano, 2003) y es entonces cuando cobra fuerza la concepción del bienestar subjetivo, la que seguramente mayor respaldo empírico ha recibido, lo cual ha permitido identificar la estructura interna de dicho constructo, diferenciando claramente tres componentes: satisfacción con la vida (Dienner, 1994; Atienza, Pons, Balaguer y García-Merita, 2000) y felicidad o balance global entre los afectos positivos y negativos (Bradburn, 1969; Argyle, 1992).

La manera de definir y medir la satisfacción con la vida también ha sufrido variaciones históricas; frente a una primera concepción como calidad de vida, que trata de buscar indicadores de la presencia de condiciones consideradas necesarias para una buena vida (Andrews y Whitey, 1976; Campbell, Converse y Rodgers, 1976), una segunda lo entiende como una valoración más subjetiva de cómo es el vivir bien o la calidad de vida "realizada" (Veenhoven, 1994) o como un elemento incluido en el dominio hedonístico (Grant, Wardle y Steptoe, 2009). Sin embargo la mayoría de las aproximaciones a la satisfacción con la vida han venido del modelo presentado por Diener y sus colaboradores. 
Por otro lado, en la Teoría de la Autodeterminación (SDT) el bienestar psicológico se considera como un funcionamiento psicológico vital basado en experiencias positivas y saludables, y en un sentido del yo congruente e integrado (Deci y Ryan, 1985; Pavot, Diener, Colvin y Sandvik, 1991; Ryan y Deci, 2001). Esta teoría maneja como indicadores de bienestar psicológico medidas de autoestima, satisfacción con la vida y vitalidad subjetiva. Por lo tanto, se encuentran asociaciones entre la satisfacción con la vida y autoconcepto-autoestima, al considerarse ambos participantes del bienestar psicológico del sujeto, ya que es visto como un indicador global crítico del sistema del yo (self-system) (Marsh, 1997). De hecho, la evidencia empírica sugiere que el autoconcepto modera las relaciones entre el estrés y la enfermedad (Rector y Roger, 1996).

Durante años se entiende el autoconcepto como un constructo unidimensional; sin embargo, en la actualidad se sostiene que los individuos generan percepciones de sí mismos muy diferentes en distintos aspectos de sus vidas (familiar, social, emocional, físico...). A partir de la década de los setenta se proponen diversos modelos del autoconcepto, entre los que destaca el de Shavelson, Hubner y Stanton (1976), que propone la condición jerárquica y multidimensional. Este modelo ha creado a partir de esta fecha una gran evidencia empírica en los distintos dominios en los que se estructura el autoconcepto.

Un dominio del autoconcepto con el que establece altas y consistentes relaciones es el autoconcepto físico (Fox, 1997; Goñi, Rodríguez y Ruiz de Azúa, 2004). Incluso, el propio autoconcepto físico conlleva importantes propiedades de bienestar psicológico. Por ejemplo, el autoconcepto físico ha mostrado relaciones con diversos indicadores de bienestar, tales como, afecto positivo y ajuste emocional, incluso cuando el autoconcepto global y la deseabilidad social ha sido estadísticamente controlada (Sonstroem y Potts, 1996; Van de Vliet et al., 2002)

Existe una evidencia acumulada de que el bienestar psicológico está asociado con comportamientos saludables. Entre los comportamientos más saludables, la actividad física está estrechamente relacionada con una mejora de la calidad o satisfacción con la vida de los individuos que la practican (McAuley et al., 2006; Rejeski y Mihalko, 2001) y con la mejora del autoconcepto físico (Fox, 2000; Infante y Zulaika, 2008). Está comprobado que las autopercepciones físicas son mejoradas a través de la participación en actividades físicas. De hecho, las autopercepciones físicas correlacionan consistentemente con la actividad física desde la adolescencia en adelante (Fox, 1997). Incluso las mejoras de las autopercepciones físicas obtenidas mediante la aplicación de programas de intervención, basados en la práctica de actividad física, pueden llegar a alcanzar el nivel más alto de la jerarquía del autoconcepto (Fox, 2000; Taylor y Fox, 2005; Infante y Zulaika, 2008). 
Se han testado diferentes modelos que explican las relaciones entre la actividad física y la calidad de vida y el que mejores índices aporta es el representado por la teoría cognitivo social (McAuley et al., 2006). Este modelo adopta la posición de Diener (1984) con respecto a la satisfacción con la vida como un constructo global que refleja un juicio cognitivo de la vida del individuo. En este modelo la aparición de un constructo intermedio, como son las autoeficacias, ha sido una constante en el estudio de la actividad física con respecto a otros constructos psicológicos. Este constructo ha ofrecido luces y sombras en la investigación de estas variables psicológicas, ya que en el caso de la satisfacción con la vida ha resultado exitoso (White, Wójcicki y McAuley, 2009), mientras que en el contexto de la autoestima los resultados no han sido tan esclarecedores (McAuley et al., 2005). Por lo tanto, aunque las autoeficacias parecen un constructo intermedio interesante en este estudio no se van a tener en cuenta debido a los distintos resultados a los que han concluido distintas investigaciones.

El presente estudio tiene como objeto analizar la asociación entre dos indicadores del bienestar psicológico (la satisfacción con la vida y el autoconcepto físico) y la práctica de actividad físico-deportiva en una muestra de adultos de edades comprendidas entre los 23 y los 64 años. Pocos esfuerzos se han realizado en determinar si otros factores psicosociales acompañan a la práctica de actividad física (White et al., 2009). Además, tampoco se entiende muy bien de qué manera influye la actividad física en la calidad de vida. El objetivo de este estudio se basa en la evidencia de que la actividad física está relacionada con la mejora de las variables psicológicas estudiadas.

\section{METODO}

\section{Hipótesis}

1. Los sujetos con puntuaciones elevadas en autoconcepto físico valorarán su vida de manera más satisfactoria que los sujetos con puntuaciones inferiores.

2. Los individuos sedentarios obtendrán puntuaciones inferiores en satisfacción con la vida que los individuos más activos.

3. El autoconcepto físico tendrá un mayor peso explicativo sobre la satisfacción con la vida que la actividad física.

\section{Participantes}

Participan en esta investigación un total de 408 personas de edades comprendidas entre los 23 y los 64 años $(M=45.41 ; D T=13.41)$. Los porcentajes referentes al sexo de la muestra revelan un cierto desequilibrio en favor de las mujeres (Tabla 1). Quienes cumplimentaron los cuestionarios pertenecen a grupos de educación no formal (Escuela de Artes y Oficios, centro de EPA -Enseñanza Permanente de Adultos- y centros deportivos privados) de la ciudad de Vitoria-Gasteiz. El número total de participantes no es igual en las dos variables de agrupación debido a que no todos los sujetos que rellenaron el cuestionario contestan a todas las preguntas. 
Tabla 1. Participantes en la investigación

\begin{tabular}{cccccc}
\hline & \multicolumn{2}{c}{ SEXO } & \multicolumn{3}{c}{ METs } \\
\hline Categoría & H & & M & Sedentarios & Activos \\
\hline Frecuencia & 163 & & 245 & 145 & 242 \\
\hline Porcentaje & $40 \%$ & & $60 \%$ & $37.5 \%$ & $62.5 \%$ \\
\hline TOTAL & & 408 & & & 387 \\
\hline
\end{tabular}

\section{Variables e instrumentos}

La satisfacción con la vida hace referencia a la satisfacción global con los diferentes ámbitos y condiciones de vida, a los objetivos planeados y conseguidos, y a los hechos de vida pasados. Para su medida se utiliza el cuestionario de Diener, Emmons, Larsen y Griffin (1985) Satisfaction With Life Scale (SWLS) traducido al castellano por Atienza et al. (2000). Tanto en la versión original como en la traducción, las propiedades psicométricas del instrumento son muy aceptables. La versión traducida y utilizada en este estudio ofrece una consistencia interna de .82 y el análisis de componentes principales consta de un único factor, que explica el $53.7 \%$ de la varianza. Las opciones de respuesta abarcan desde En total desacuerdo, valorado con un 1, hasta Totalmente de acuerdo, puntuado con un 7.

El autoconcepto físico fue medido empleando el Cuestionario de Autoconcepto Físico (CAF), de Goñi et al. (2006), que consta de 36 ítems (6 por cada escala) destinados a medir los siguientes componentes del autoconcepto: Habilidad física, Condición física, Atractivo físico, Fuerza, Autoconcepto físico general y Autoconcepto General. Todas las escalas ofrecen un nivel alto de fiabilidad, cuyos valores oscilan entre el .80 y el .88. El formato de respuesta se divide en cinco alternativas en una escala tipo Likert, de modo que una mayor puntuación indicaría un autoconcepto físico más elevado. El cuestionario ha mostrado sensibilidad para discriminar entre las respuestas de personas de distintas edades y entre hombres y mujeres. En referencia a la práctica deportiva se ha comprobado que es capaz de discriminar la frecuencia y el tipo de organización de la actividad físico-deportiva en la que se participa.

En este estudio se habla de actividad físico-deportiva, término que engloba tanto al ejercicio físico como al deporte. Se dejan de lado las actividades físicas cotidianas, tales como las domésticas y laborales. Para medir la actividad físicodeportiva realizada se preguntó a los participantes acerca de la frecuencia, duración y modalidad de actividad en el tiempo libre en la que habían estado implicados en los últimos 15 días. A cada modalidad de actividad físico-deportiva se le asignó un valor de intensidad (MET sistema de equivalente metabólico) basado en el trabajo de Ainsworth et al. (2000) y siguiendo la siguiente formula $($ MET/2sem $=$ Frecuencia $\times$ Duración $\times$ Valor de la actividad físico-deportiva). Una vez calculado el índice energético y siguiendo los criterios establecidos en la Encuesta de Salud de la Comunidad Autonómica del País Vasco del 2007, se dividió a los participantes en 4 cuatro 
categorías: los sedentarios, con un gasto energético menor a 1.250 METs; los moderadamente activos entre 1.250 y 2.999 METs; los activos entre 3.000 y 5.499 METs; y los muy activos con un gasto energético superior a 5.500 METs.

\section{Procedimiento}

Se contactó con los responsables de los centros de pertenencia de los participantes (Escuela de Artes y Oficios, centro de EPA) al objeto de darles a conocer los objetivos de la investigación y solicitar autorización para llevarla a cabo; asimismo con la colaboración de monitores de academias de danza y de gimnasios deportivos, se distribuyeron cuestionarios entre asistentes a estas actividades. De otra parte, para reducir el efecto de deseabilidad social, se cumplimentaron los cuestionarios de forma anónima; y con el objeto de reducir el efecto fatiga, los cuestionarios se presentaron en diferentes colores y formatos.

\section{RESULTADOS}

Con el objeto de estudiar la relación existente entre las variables de interés de este estudio, léase actividad físico-deportiva, autoconcepto físico y satisfacción con la vida, se realizan diferentes tipos de cálculos: análisis de la correlación entre dichas variables, ANOVA para examinar la variación de la satisfacción con la vida en función de diferentes niveles de autoconcepto, y análisis de la varianza univariado.

En el primer caso, se opta por hacer un análisis básico de correlación bivariada entre las tres variables, incluidas las dimensiones del autoconcepto físico. Los datos muestran que la relación del autoconcepto físico con respecto a la práctica de actividad físico-deportiva y la satisfacción con la vida es significativa, a excepción de la dimensión habilidad que presenta una correlación de .049 con satisfacción con la vida. El resto de índices se encuentran entre un mínimo de $.151(p<.01)$ (atractivo y METs) y un máximo de .372 ( $p<.000)$ (condición y METs). Por el contrario, la relación entre la actividad físico-deportiva y la satisfacción con la vida es positiva pero dista de ser significativa $(r=.017)$.

Para profundizar más en la naturaleza de estas relaciones se lleva a cabo, en primer lugar, una ANOVA (Tabla 2) en la que la variable dependiente es la satisfacción con la vida y la independiente el autoconcepto físico, dividido en tres niveles (bajo, medio y alto); el procedimiento de cálculo del límite de los niveles se realiza a partir de la puntuación media y la desviación típica de cada dimensión.

Los resultados indican que los sujetos con autoconcepto alto obtienen también puntuaciones superiores en satisfacción con la vida a las de los sujetos de nivel medio o bajo. Aunque esta tendencia creciente no es significativa en el caso de habilidad y condición, ocurre en todas las dimensiones del autoconcepto físico, e incluso la dimensión de autoconcepto general. 
Tabla 2. Satisfacción con la vida en función del autoconcepto físico

\begin{tabular}{|c|c|c|c|c|c|c|}
\hline & & $N$ & $M$ & $S D$ & $F$ & $p$ \\
\hline \multirow{3}{*}{ HAB } & Baja & 63 & 23.83 & 5.36 & \multirow{3}{*}{.604} & \multirow{3}{*}{.547} \\
\hline & Media & 253 & 24.52 & 4.88 & & \\
\hline & Alta & 67 & 24.81 & 6.89 & & \\
\hline \multirow{3}{*}{$\mathrm{CON}$} & Baja & 69 & 23.55 & 5.85 & \multirow{3}{*}{2.505} & \multirow{3}{*}{.083} \\
\hline & Media & 246 & 24.38 & 5.00 & & \\
\hline & Alta & 71 & 25.55 & 5.91 & & \\
\hline \multirow{3}{*}{ ATR } & Baja & 60 & 22.03 & 5.38 & \multirow{3}{*}{11.291} & \multirow{3}{*}{$.000 * * *$} \\
\hline & Media & 274 & 24.55 & 5.04 & & \\
\hline & Alta & 52 & 26.69 & 5.91 & & \\
\hline \multirow{3}{*}{ FUE } & Baja & 70 & 23.10 & 5.73 & \multirow{3}{*}{4.396} & \multirow{3}{*}{$.013^{*}$} \\
\hline & Media & 248 & 24.47 & 4.94 & & \\
\hline & Alta & 68 & 25.78 & 6.13 & & \\
\hline \multirow{3}{*}{$\mathrm{AFG}$} & Baja & 64 & 21.77 & 5.77 & \multirow{3}{*}{16.675} & \multirow{3}{*}{$.000 * * *$} \\
\hline & Media & 250 & 24.44 & 5.06 & & \\
\hline & Alta & 72 & 26.87 & 4.88 & & \\
\hline \multirow{3}{*}{$\mathrm{AG}$} & Baja & 65 & 19.43 & 5.62 & \multirow{3}{*}{56.457} & \multirow{3}{*}{$.000 * * *$} \\
\hline & Media & 263 & 24.86 & 4.70 & & \\
\hline & Alta & 58 & 28.24 & 3.57 & & \\
\hline
\end{tabular}

$* \mathrm{p}<.05 ; * * * \mathrm{p}<.001$

Se procede, en segundo lugar, a calcular el modelo general univariante tomando nuevamente como variable dependiente la satisfacción con la vida; se comprueba la significatividad de los efectos principales del autoconcepto físico y de la actividad físico-deportiva, así como la de la interacción de ambos factores fijos. Los análisis se llevan a cabo para cada una de las dimensiones del autoconcepto físico, pero aquí (Tabla 3) tan sólo se presentan los resultados correspondientes al dominio general de autoconcepto físico.

Los resultados indican que la interacción de ambas variables no está relacionada significativamente con una variación en la puntuación de la satisfacción con la vida, siendo únicamente significativo el peso de la variable autoconcepto físico. Es decir, que una mayor o menor satisfacción con la vida está vinculada al autoconcepto físico, independientemente de la práctica de actividad físico-deportiva.

Tabla 3. Satisfacción con la vida en función del autoconcepto físico y la práctica de actividad físico-deportiva

\begin{tabular}{|c|c|c|c|c|c|c|c|}
\hline & & & \multicolumn{4}{|c|}{ Actividad físico-deportiva } & \\
\hline & & & 1 & 2 & 3 & 4 & \\
\hline \multirow{6}{*}{ 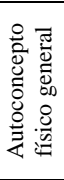 } & \multirow{2}{*}{ Bajo } & $M$ & 22.67 & 21.20 & 20.00 & 22.67 & Actividad físico-deportiva $(F=1059)$ \\
\hline & & $D T$ & 4.88 & 6.27 & 7.68 & 4.62 & $.366 / \eta^{2}=.009$ \\
\hline & \multirow{2}{*}{ Medio } & $M$ & 24.70 & 24.23 & 23.31 & 24.92 & Autoconcepto físico $(F=11.084)$ \\
\hline & & $D T$ & 4.74 & 5.04 & 4.83 & 5.16 & $.000 * * * / \eta^{2}=.059$ \\
\hline & \multirow{2}{*}{ Alto } & $M$ & 26.37 & 27.56 & 25.67 & 27.23 & $A F D * A F(F=.281) .946 / \eta^{2}=.005$ \\
\hline & & $D T$ & 5.49 & 3.46 & 5.95 & 4.88 & \\
\hline
\end{tabular}

La capacidad explicativa de ésta segunda variable, aunque no excesivamente elevada, tal y como indica la puntuación .059 en el $\eta^{2}$, es mayor a la de la actividad físico-deportiva. Este resultado es una constante en los análisis realizados con todas las dimensiones del autoconcepto físico. Incluso en los casos de habilidad y condición, en 
los que tampoco el efecto de las mismas es estadísticamente significativo, su índice $\eta^{2}$ es superior al del grado de práctica de actividad físico-deportiva.

\section{DISCUSION}

La satisfacción con la vida y el autoconcepto son considerados como componentes del bienestar psicológico de la persona, ítem de indudable interés en las sociedades desarrolladas que buscan una constante mejora, aunque no siempre con resultados satisfactorios. Dentro del autoconcepto, existen diferentes dominios que afectarían hipotéticamente a dicho bienestar, ocupando el autoconcepto físico un lugar privilegiado en la valoración que el sujeto hace de sí mismo.

Los resultados de este estudio muestran evidencias de que ambos constructos, satisfacción con la vida y autoconcepto físico, son realidades estrechamente vinculadas; las personas con mayor nivel de autoconcepto valoran su vida de manera más positiva (Taylor y Fox, 2005). Este patrón de variación es igual en el caso de la relación entre el autoconcepto físico y la práctica físico-deportiva, tal y como han demostrado estudios previos (Fox, 2000; Taylor y Fox, 2005; Infante y Zulaika, 2008). Lo sorprendente de este estudio es que las dimensiones de habilidad y condición física no se muestran relacionadas de manera tan intensa como la fuerza y el atractivo físico; en las sociedades occidentales de manera especial, la apariencia y la fuerza física, también como elemento de valor en la imagen del hombre, pueden ser factores más determinantes de la satisfacción física que cualquiera de las demás dimensiones del autoconcepto (Humberson y Hugues, 1987; Rodríguez y Fernández, 2005).

Por el contrario, la relación entre la práctica de actividad y la satisfacción con la vida no ha resultado estadísticamente significativa, si bien también es positiva. Este dato no conviene plenamente con lo hallado hasta el momento (Rejeski y Mihalko, 2001; McAuley, et al., 2006), aunque las distintas concepciones de la satisfacción con la vida pueden ser la explicación de estos resultados divergentes; tampoco coincide con algunos estudios que afirman que la actividad física contribuye al bienestar psicológico (Biddle et al., 2000; Goñi y Rodríguez, 2004; Thórgersen-Ntoumani, Fox y Ntoumanis, 2005). El hecho es que en la explicación de la variabilidad de las puntuaciones en satisfacción con la vida el autoconcepto físico, al menos algunas de sus dimensiones, tiene un peso importante, pero no ocurre así con el nivel de actividad física del sujeto.

Por lo tanto, la satisfacción con la vida es superior en quienes tienen una percepción del ámbito físico propio más positiva, independientemente de su nivel de práctica. A su vez, el autoconcepto físico es superior en quienes practican actividad con mayor frecuencia, por lo que parece lógico pensar que la práctica de actividad físico deportiva esté relacionada indirectamente con la satisfacción con la vida a través del autoconcepto físico (White et al, 2009), aunque todavía no sé conoce con exactitud 
cómo es el mecanismo por el que se relacionan estas variables (Lotan, Merrick y Carmeli, 2005; Thórgersen-Ntoumani et al., 2005; Rodríguez, 2008).

\section{Agradecimientos}

Este artículo es resultado del proyecto de EDU2009-10102 (subprograma EDUC) subvencionado por el MICINN.

\section{REFERENCIAS}

Ainsworth et al. (2000). Compendium of physical activities: an update of activity codes and MET intensities. Medicine and Science in Sports and Exercise, 32(9), Suppl., S498-S516.

Andrews, M. y Withey, S. (1974). Social indicators of well-being: America's perception of life quality: Results from several national surveys. Social Indicators Research, 1, 1-26.

Argyle, M. (1992). La psicología de la felicidad. Madrid: Alianza.

Atienza, F., Pons, D., Balaguer, I. y García-Merita, M. (2000). Propiedades psicométricas de la Escala de Satisfacción con la Vida en adolescentes. Psicothema, 12, 314-319.

Biddle, S., Fox, K. y Boutcher, S.H. (2000). Physical activity and psychological well-being. London: Routledge.

Bradburn, N. (1969). The structure of psychological well-being. Chicago: Aldine.

Campbell, A., Converse, P. y Rodgers, W. (1976). The quality of American life: Perceptions, evaluations and satisfactions. New York: Russell Sage Foundation.

Cuadra, H. y Florenzano, R. (2003). El bienestar subjetivo: Hacia una psicología positiva. Revista de Psicología, 12, 83-96.

Deci, E. y Ryan, R. (1985). Intrinsic motivation and self-determination in human behaviour. New York: Plenum Press.

Diener, E. (1984). Subjective well-being. Psychological Bulleting, 95, 542-575.

Diener, E. (1994). El bienestar subjetivo. Intervención Psicosocial, 3, 67-113.

Diener, E. (2000). Subjective well-being: The science of happiness and a proposal for a national index. American Psychologist, 55, 34-43.

Fox, K. (1997). The physical self and processes in self-esteem development. En K.R. Fox (Ed), The physical self: From motivation to well-being (pp. 111-139). Champaign, IL: Human Kinetics.

Fox, K. (2000). The effects of exercise on self-perceptions and self-esteem. En S.J.H. Biddle, K. R. Fox y S.H. Boutcher (Eds.), Physical activity and Psychological well-being (pp. 88-117). London: Routledge.

Goñi, A., Ruiz de Azúa, S. y Rodríguez, A. (2004). Deporte y autoconcepto físico en la preadolescencia. APUNTS. Educación Física y Deportes, 77, 18-24.

Grant, N., Wardle, J. y Steptoe, A. (2009). The relationship between life satisfaction and health behaviour: A cross-cultural analysis of youth adults. International Journal of Behaviour Medicine, 16, 259-268.

Infante, G. y Zulaika, L. (2008). Actividad física y autoconcepto físico. En A. Goñi (Coord.), El autoconcepto físico (pp. 125-153). Madrid: Pirámide.

Lotan, M., Merrick, J. y Carmeli, E. (2005). Physical activity in adolescence. A review with clinical suggestions. International Journal of Adolescent Medicine and Health, 17(1), 13-21.

Marsh, H. (1997). The measurement of physical self-concept: a construct validation approach. En K.R. Fox (Ed.), The physical self. From motivation to well-being (pp. 27-58). Champaign: Human Kinetics. 
McAuley, E., Elavsky, S., Motl, R., Konopack, J., Hu, L. y Marquez, D. (2005). Physical activity, self-efficacy and self-esteem: Longitudinal relationships in older adults. Journal of Gerontology, 60(5), 268-275.

McAuley, E., Konopack, J., Morris, K., Motl, R., Hu, L., Doerksen, S. y Rosengren, K. (2006). Physical activity and functional limitations in older women :influence of self-efficacy. Journal of Gerontology: Psychological Sciences, 61(5), 270-277.

Pavot, W., Diener, E., Colvin, C. y Sandvik, E. (1991). Futher validation of the Satisfaction With the Life Scale: Evidence for the cross-method convergence of well-being. Social Indicators Research, 28, 1-20.

Pressman, S. y Cohen, S. (2005). Does positive affect influence health? Psychological Bulletin, 131, 925-971.

Rector, N. y Roger, D. (1996). Cognitive style and well-being: A prospective examination. Personality and Individual Differences, 21, 663-674.

Rejeski, W. y Mihalko, S. (2001). Physical activity and quality of life in older adults. Journal of Gerontology: Psychological Sciences, 56(A), 23-35.

Rodríguez, A. (2008). Bienestar y malestar psicológico en la adolescencia. Leioa: UPV/EHU.

Rodríguez, A. y Fernández, A. (2005). Los componentes del bienestar psicológico y el autoconcepto físico de los adolescentes. En M.I. Fajardo, F. Vicente, A. Ventura, I. Ruiz, y J.A. del Barrio (Eds.), Aportaciones psicológicas y mundo actual. Dando respuestas (pp. 465-480). Badajoz: Psicoex.

Ryan, R. y Deci, E. (2001a). On happiness and human potentials: A review if research on hedonic and eudaimonic well-being. Annual Review of Psychologist, 52(1), 68-78.

Ryan, R. y Deci, E. (2001b). To be happy or to be self-fulfilled: A review of research on hedonic and eudaemonic well-being. En S. Fiske (Ed.), Annual Review of Psychology, 52 (pp. 141-166). Palo Alto: Annual Reviews.

Shavelson, R., Hubner, J. y Stanton, J. (1976). Self concept: Validation of construct interpretations. Review of Educational Research, 46, 407-441.

Sonstroem, R. y Potts, S. (1996). Life adjustment correlates of physical self-concepts. Medicine and Science in Sports and Exercise, 21, 329-337.

Taylor, A. y Fox, K. (2005). Effectiveness of a primary care exercise referral intervention for changing physical self-perceptions over nine months. Health Psychology, 24(1), 11-21.

Thórgersen-Ntoumani, C., Fox, K.R. y Ntoumanis, N. (2005). Relationship between exercise and three components of mental well-being in corporate employees. Psychology of Sport and Exercise, 6, 609-627.

Umberson, D. y Hughes, M. (1987). The impact of physical attractiveness on achievement and psychological well-being. Social Psychology Quarterly, 50, 227-236.

Van de Vliet, P., Knapen, J., Onghena, P., Fox, K., Van Coppenolle, H., David, A., Pieters, G. y Peuskens, J. (2002). Assessment of physical self-perceptions in normal Flemish adults versus depressed psychiatric patients. Personality and Individual Differences, 32, 855-863.

Veenhoven, R. (1994). El estudio de la satisfacción con la vida. Intervención Psicosocial. Revista sobre Igualdad y Calidad de Vida, 3, 87-116.

White, M., Wójcicki, R. y McAuley, E. (2009). Physical activity and quality of life in community dwelling older adults. Health and Quality of Life Outcomes, 7(10). 
GOÑI e INFANTE. Actividad físico-deportiva, autoconcepto y satisfacción

Recepción Modificaciones: 19 de enero de 2010 Aceptado: 22 de enero de 2010 